\title{
Application of Mathematical Morphology in Simulation of Electrical Properties
}

\author{
Qiang fu Kong \& Hua chao Sun \\ PetroChina Research Institute of Petroleum Exploration \& Development, Beijing
}

\begin{abstract}
This paper applied the mathematical morphology method to the digital core electrical simulation, used opening operation in mathematical morphological to characterized fluid distribution under different saturation in pore space, laid the foundation for the further study on the variation of resistance increase index, and also analyzed some disadvantages of mathematical morphology method in the simulation of electrical properties.
\end{abstract}

KEYWORD: Mathematic morphology; Open operation; numerical simulation of electrical properties; the fluid distribution

\section{INTRODUCTION}

As we all known that rock electrical data plays an important role in logging interpretation, reservoir evaluation and prediction in the reserves. While the core flow distribution characteristics is difficult to quantitative calculation, control and observation in rock physics experiment. The numerical simulation based on digital core of rock electrical properties can make up for the shortage. In the electrical numerical simulation for a single phase fluid saturated rocks, the fluid in pore space without special treatment, respectively. But for two phase fluid saturated rocks, the electrical properties of the rock have much link with the fluid distribution. Under different water saturation, the pore fluid distribution is affected by many micro factors, so based on the digital core, how to determine the oil and water distribution under different water saturation is the top issue that the numerical simulation of rock electrical properties were faced with. After determined the threedimensional digital core pore fluid distribution, use numerical simulation methods to calculate the resistivity of the rock, and study the law of rock resistivity index change.

The introduction of mathematical morphology method provide a train of thoughts for numerical simulation of electrical properties pore under different saturation fluid distribution and laid the foundation to investigate the law of resistivity index change. This article is on the basis of core CT scan images, by processing core pore space in binary images with mathematical morphology method, finally characterized the entire three-dimensional digital core fluid distribution characteristics of the pore space.

\section{MATHEMATICAL MORPHOLOGY}

Image morphology is a generalization and development of mathematical morphology, the mathematical morphology is closely associated with integral. Its history can goes back to the 19th century by Euler, Steiner, Crofton and at the beginning of this century by Minkowski. In 1964, France G. atheron and J.Serra on the basis of integral research results, founded the mathematical morphology, then developed image processing system (such as Leitz, etc.) which was based on mathematical morphology. That the Image Analysis and Mathematical Morphology was published in 1982 monograph is an important milestone in the development of Mathematical Morphology. It shows that mathematical morphology tends to be more perfect in theory and application. In 1986 CVGIP published a special issue mathematical morphology [1-3].

Mathematical morphology method, based on the form of image, is a kind of mathematical tools to analysis the images and belongs to the nonlinear signal processing method. Its basic idea is to use certain forms of structural elements to measure or extract image of the corresponding shape to achieve the aim of image analysis and recognition, research is focused on image geometric structure, mathematics is based on set theory. Nowadays with the development of computational mathematics morphology has been widely used in computer 
language identification, microscopic image analysis, medical image processing, industrial inspection and robot vision.

Mathematical morphology is composed of a set of algebraic operation. There are four basic operations: inflation (or expansion), (corrosion) or erosion, opening operation and closing operation. Corrosion operation and expansion operation is the foundation of the mathematical morphology processes, many mathematical morphology algorithm are based on these two basic operations.

\subsection{Corrosion operation}

Set A and B is the set of $Z^{2}, \emptyset$ as the empty set, the erosion of $A$ by $B$, to remember $A \ominus B$, $\ominus$ for corrosion operator. Corrosion operation is defined as

$$
\mathrm{A} \ominus \mathrm{B}=\left\{\mathrm{x} \mid(B)_{x} \subseteq A\right\}
$$

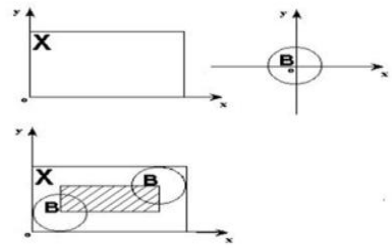

Figure 1. corrosion operation schematic diagram.

Corrosion arithmetic is used to remove isolated point in image and burr, cut area boundary, increase the internal hole, and disconnect the links between certain areas.

\subsection{Expansion operation}

Set A and B is the set of $Z^{2}, \emptyset$ as the empty set, the expansion of $A$ by $B$, to remember $A \oplus B, \oplus$ for expansion operator. Expansion operation is defined as

$$
\mathrm{A} \oplus \mathrm{B}=\left\{\mathrm{x} \mid(\widehat{B})_{x} \cap A \neq \emptyset\right\}
$$

The formula show that the expansion of the operation process is B set to mapping first do about its origin, then translation $\mathrm{x}$. The expansion of the A is $\mathrm{B}$ operation is $\widehat{B}$ after all $\mathrm{x}$ translation, there are at least a nonzero common elements and set A. As other algorithm of mathematical morphology, set B is referred to as structural elements in the expansion operation. Figure 2 shows an expansion process, the shadow part of the figure for inflation is the results after expansion.

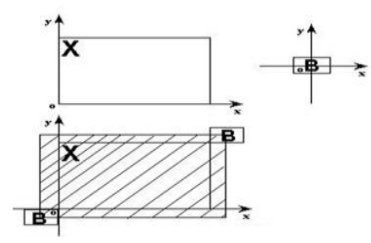

Figure 2. expansion operation schematic diagram
The role of the expansion operation is to increase the edge of the image area and decrease the area of the hole.

\subsection{Open operation}

Take structure element B as open operation, record for $\mathrm{A} \circ \mathrm{B}$, defined as

$$
\mathrm{A} \circ \mathrm{B}=(A \ominus B) \oplus \mathrm{B}
$$

In other words, set $\mathrm{A}$ by set $\mathrm{B}$ to do open operation is $\mathrm{B}$ to be the result after corrosion of the expansion.

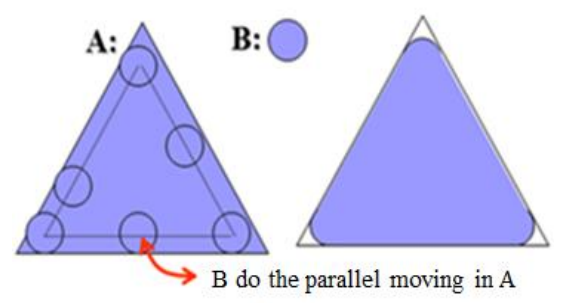

Figure 3. open operation schematic diagram.

The function of Opening operation in mathematical morphology has a simple geometrical interpretation. Assume the disc structure element B as a plane "rolling ball". The boundary of A $\circ \mathrm{B}$ is the collection of $\mathrm{B}$ in $\mathrm{A}$ scroll to the most distant from the boundary B. All the outward in the set A prominent part remains the same. Outstanding and cannot accommodate structural elements of the part is removed.

Open operations under the condition of the general location and shape unchanged eliminate smaller than structural elements of a particular image details, smoothing the contours of the image, expanding gap, separated by thin lines connected objects, eliminate the tiny bumps and isolated points.

\subsection{Close operation}

Take structure element B to do closed operation, record for $\mathrm{A} \circ \mathrm{B}$, defined as

$$
\mathrm{A} \cdot \mathrm{B}=(A \oplus B) \Theta \mathrm{B}
$$

In other words, set $\mathrm{A}$ by set $\mathrm{B}$ to do open operation is $\mathrm{B}$ to be the result after expansion of the corrosion.

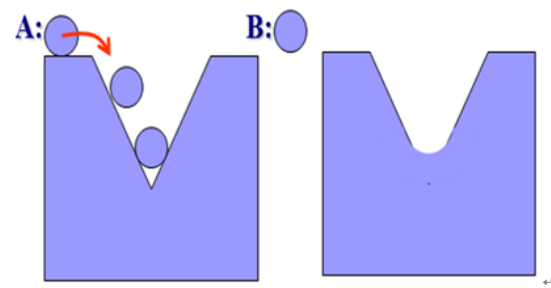

Figure 4. closed operation schematic diagram. 
Closed operation also has the similar explanation as open operation. Use a rolling ball as an example again, but the difference is that rolling the ball outside the boundary. All incoming highlights have been smooth, but not for exterior impact. Recessed part of the left of set A was sharply reduced.

Closed operations under the condition of the general location and shape unchanged fills the hole in the target, connected to the adjacent smaller goals and breakpoints, from the external boundary, filling in the image smooth aperture, fusion of adjacent images small pieces.

\section{OIL AND WATER DISTRIBUTION UNDER DIFFERENT SATURATION OF PORE SPACE}

If only considering pore space and skeleton, 3D digital core can be regarded as the 3D binary digital images, with 1 represents the skeleton, 0 indicates the pore. So the mathematical morphology method was suitable for 3D digital core. In order to display the result of mathematical morphology operations more intuitive, use a 3D digital core slice (twodimensional scanning image) as an example to introduce the image corrosion operation, expansion operation and open operation.

\subsection{Two-dimensional medium image processing}

Figure 5 is the core scanning the two-dimensional image, white represents rock porosity, size is $2049 *$ 1362 pixels, and the black represents rock particles. In original image, with 1 indicates the skeleton and pore space was expressed as zero. Therefore mathematical morphology operations are all operated on porous media pore space. Selected the radius $\mathrm{R}$ to 15 pixels circle as structural elements, to do the pore space in figure 5 (a) (white area) for corrosion, expansion, and open operation, the result of the operation as shown in figure 5 (b) to (d) is shown in figure 5 , the red part of the figure represent the results of pore space after the corresponding operation, can be seen that expansion operation enlarge images, corrosion computing shrinkage image. Open operation can be understood as structural elements in the X B formed within the boundary of the scroll can achieve space, as shown in figure $5(\mathrm{~d})$ in the red areas. At the same time the definition of open operation pore space open computing results for all radius is the larger space than the pore space $\mathrm{R}$.

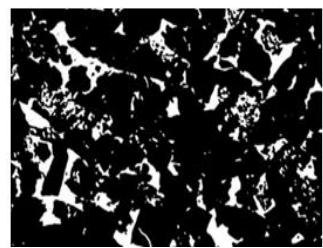

(a) The original image

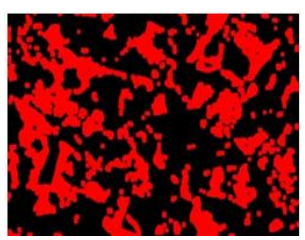

(c) Expansion operation

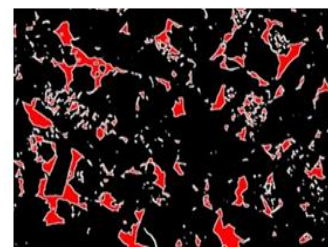

(b) Corrosion operation

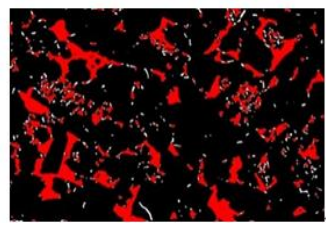

(d) Open operation
Figure 5. Porous media two-dimensional image corrosion, expansion and operation schematic diagram.

\subsection{Oil and water distribution characterization}

Assume the biggest pore radius in the rock pore space is $\mathrm{R}$ max. Take structural elements radius as $\mathrm{R} \_$max, the result of opening function through the analysis above is the maximum pore in the pore space. When the radius of structural elements from big to small changes in turn, rock pore space to do the open operations, respectively. It can be seen that with the decrease of the radius of structural elements, the result of the pore space open operation characterization of the pore space according to the size of the pore radius, in turn, increases, as shown in figure 6. To illustrate the results of twodimensional pore space open operation, we do not take into account the connection between the intrusive facies. Figure 6 (a) to figure 6 (d), radius of structural elements from big to small changes in turn. If the results of of a pore space to do open computing which representing the characterization of oil in the process of water displacement oil, the rest of the pore space is characterization of formation water, then this process is similar to water wet rock's expulsion process. In water wet rock the non-wet phase oil in the pore space will occupied the big pore first, with the increase of displacement pressure, oil will in accordance with the sequence to intrusion from large to small pore radius. So the use of rock pore space open operation can simulate the process of water wet rock displacement, then we can further to determine pore space under different water saturation medium oil and water in the distribution of the pore space. As shown in figure 7, blue said skeleton, green water, red said oil.

That Oil wet rock under different water saturation of pore fluid distribution is different from water wet rock. For oil wet rock, oil occupied little pore in the rock pore space first, while the big pore space occupied by water. So the oil in accordance with the order of the pore radius from small to large to occupied pore space. After determined the fluid distribution in pore space of oil wet rock under different water saturation, and still uses the 
simulation method of water wet rock, structural elements radius from big to small changes in turn. But fluid distribution in pore space of water wet reservoirs is exactly opposite, the result of open operation for the pore space represents the characterization of pore space was dominated by formation water, and the rest of the pore space occupied by a oil.

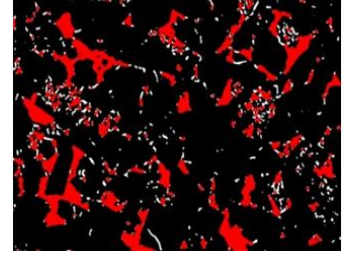

(a)structural elements radius 15

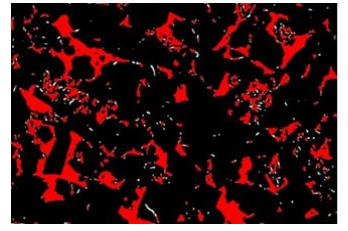

(c) structural elements radius 13

Figure 6. open operation result under different radius of structure elements
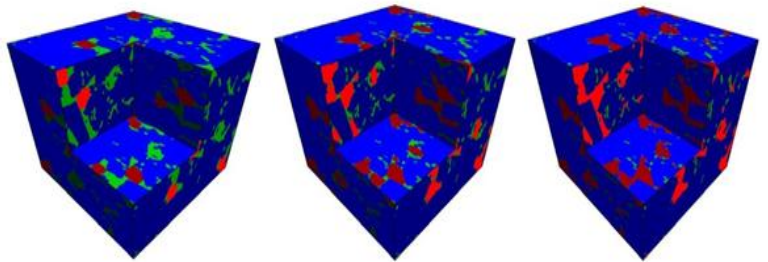

(a) $S_{w p}=84 \%$

(b) $S_{\mathrm{we}}=54 \%$

(c) $S_{w}=27 \%$.

Figure 7 pore fluid distribution under different saturation

\subsection{The problems existed}

In the method of mathematical morphology to determine the pore fluid distribution under different water saturation of pore fluid is composed of two phase: oil and formation water. In order to eliminate the influence that formation water does on the simulation results of resistivity Late, the results of numerical simulation of rock electrical properties and resistivity of formation factors was adopted as the index to describe, while in the wet rocks, pore is full of oil after the invasion, the presence of water in the pore surface membrane which provides an additional channel for the current conduction. During the high water saturation, the water film conduction effect is not obvious, but at low water saturation, the conduction function of water film can't be ignored. Within the scope of the whole water saturation to simulate rock resistivity accurately the water film conduction effect must be considered. The crux of the problem is how to represent water film in 3D digital core. In general, as a result of the water film thickness is far less than the resolution of the digital core, therefore we cannot used the outermost layers of the pore pixels which was invaded by oil phase as water film directly, this is also a difficult point of the present study; so this paper use a "equivalent treatment" tool that by using the ratio of oil phase to all the outermost pixel number multiplied by the thickness of the water film with the number of oil phase pixel number as the water film at the position between oil phase and the skeleton pixel contact position, just see fig 8 the red area; On the other hand because of the structural elements is spherical, result in we unable to handle complicated pore space, such as cracks. Also the radius of the structural elements must be an integer, so it lead to the displacement pressure cannot change continuously, therefore it can only calculate the resistivity of a finite number of water saturation [7].

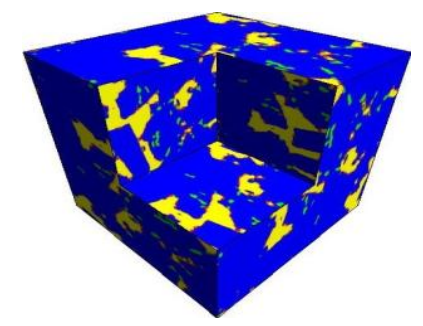

Fig 8 characterization of water film

\section{CONCLUSIONS}

In this paper, the research work mainly focused on using the mathematical morphology method to simulate the oil and water distribution of pore space, without doing the resistivity of the simulation, then point out some problems of the mathematical morphology method, so we can draw the following points:

(1) Based on the digital core, Mathematical morphology method provides a train of thought of numerical simulation of rock electrical characterization of pore fluid distribution in the space to study resistivity index change law;

(2) Mathematical morphology methods in characterization of water film and continuous saturation change was still exist certain difficulties, so further studies are needed.

\section{REFERENCES}

[1] Jia Yong Hong. Digital image process. Wuhan university press, 2005:173-175.

[2] He Ming Yi, Wei Bao Guo. Digital image process. Science press, 2007:218-226.

[3] William K Pratt. Digital image process. Machinery industry press, 2010:360-368.

[4] Zhu Hong. Digital image process. Science press, 2006:154-156.

[5] http://cmm.ensmp.fr/index_eng.html.

[6] http://www.cwi.nl/projects/morphology.

[7] Liu Xue Feng. Numerical simulation of rock electrical properties based on digital cores. Shandong Dong Ying: China University of Petroleum (east of China), 2010. 